\title{
Causes, functional outcomes and healthcare utilisation of people with cerebral palsy in Singapore
}

Zhi Min Ng, ${ }^{1}{ }_{M R C P C H}(U K)$, Jeremy B Lin, ${ }^{2}{ }_{M R C P C H}(U K)$, Poh Choo Khoo,${ }^{3}{ }_{M R C P C H}(U K)$, Victor Samuel Rajadurai, ${ }^{3}$ MRCP (UK)(Paed),

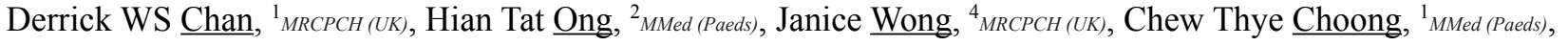
Kim Whee Lim, ${ }^{1}{ }_{M M e d}$ (Paeds), Kevin BL Lim, ${ }_{\text {FRCSEd (Orth) }}$, Tong Hong Yeo, ${ }^{1}{ }_{\text {FRCPCH-RCPCH (UK) }}$

\begin{abstract}
Introduction: A voluntary cerebral palsy (CP) registry was established in 2017 to describe the clinical characteristics and functional outcomes of CP in Singapore.

Methods: People with CP born after 1994 were recruited through KK Women's and Children's Hospital, National University Hospital and Cerebral Palsy Alliance Singapore. Patient-reported basic demographics, service utilisation and quality of life measures were collected with standardised questionnaires. Clinical information was obtained through hospital medical records.

Results: Between 1 September 2017 and 31 March 2020, 151 participants were recruited. A majority $(\mathrm{n}=135,89 \%)$ acquired $\mathrm{CP}$ in the pre/perinatal period, where prematurity $(\mathrm{n}=102,76 \%)$ and the need for emergency caesarean section $(n=68,50 \%)$ were leading risk factors. Sixteen $(11 \%)$ of the total participants had post-neonatally acquired CP. For predominant CP motor types, $109(72 \%)$ had a spastic motor type; $32 \%$ with spastic mono/hemiplegia, $41 \%$ diplegia, $6 \%$ triplegia and $21 \%$ quadriplegia. The remaining $(42,27.8 \%)$ had dyskinetic CP. Sixty-eight $(45.0 \%)$ participants suffered significant functional impairment (Gross Motor Functional Classification System levels IV-V). Most participants $(n=102,67.5 \%)$ required frequent medical follow-up ( $\geq 4$ times a year).

Conclusion: Optimisation of pre- and perinatal care to prevent and manage prematurity could reduce the burden of $\mathrm{CP}$ and their overall healthcare utilisation.
\end{abstract}

Ann Acad Med Singap 2021;50:111-8

Keywords: Cerebral palsy, functional outcomes, neonatal, registry

\section{INTRODUCTION}

Cerebral palsy (CP) describes a group of permanent, but often changing, disorders that affect movement and posture, causing activity limitation, attributed to nonprogressive disturbances that occurred in the developing fetal or infant brain. ${ }^{1}$ The birth prevalence of $\mathrm{CP}$ is estimated to be 1.4-2.2 per 1,000 in high-income countries. It is one of the most common and severe disabilities in childhood, with high individual and societal demands on health, educational and social services. ${ }^{2-4}$

Since the first population-based CP registry was set up in Denmark in 1950, over 40 registries have been established, mainly in European countries, Canada and Australia. ${ }^{5}$ These large databases provide a wealth of information on prevalence, aetiology, risk factors, temporal trends and treatment strategies. This forms a basis upon which to plan services, conduct research and act as a springboard for advances in therapeutics and rehabilitation. Historically, there has been limited data on $\mathrm{CP}$ in both Asia and in low-and middle-income countries worldwide. However, with the establishment of new surveillance programmes in the region in the last 2 decades, this is beginning to change. The Korean Database of Cerebral Palsy was developed in 2009 and a CP

\footnotetext{
${ }^{1}$ Department of Paediatrics, KK Women's and Children's Hospital, Singapore

${ }^{2}$ Department of Paediatrics, Yong Loo Lin School of Medicine, National University of Singapore and Khoo Teck Puat-National University Children's Medical Institute, National University Health System, Singapore

${ }^{3}$ Department of Neonatology, KK Women's and Children's Hospital, Singapore

${ }^{4}$ Dr Janice Paediatric Centre, Singapore

${ }^{5}$ Department of Orthopaedic Surgery, KK Women's and Children's Hospital, Singapore

Correspondence: Dr Zhi Min Ng, Department of Paediatrics, KK Women's and Children's Hospital, 100 Bukit Timah Road, Singapore 229899. Email: ng.zhi. min@singhealth.com.sg

Dr Jeremy B Lin, NUHS Tower Block Level 12, 1E Kent Ridge Road, Singapore 11922.

Email: jeremy_lin@nuhs.edu.sg
} 
registration system has been developed in Japan. ${ }^{6,7}$ Epidemiology of CP has also been studied in different parts of China, Pakistan and Nepal based on populationbased surveys, local disability registration systems and hospital-based studies. ${ }^{8-12}$ New CP registers have also been formed in Bangladesh, Sri Lanka, Vietnam and other Asian countries., ${ }^{43,14}$ These CP registers are beginning to identify both the birth prevalence of $\mathrm{CP}$ in these regions, which is essential to understand service requirements, and also to identify region-specific opportunities for prevention. Singapore, despite being a developed country in Asia, lacks comprehensive data on the burden of disease for this common lifelong neurological condition.

The Cerebral Palsy Registry in Singapore (SingCPR) was established in September 2017. The key objectives of the Registry are: (1) to determine the clinical characteristics and functional outcomes of people with $\mathrm{CP}$ in Singapore; (2) to assist in planning, development and provision of resources and services for CP locally; and (3) to identify areas for further research to improve clinical outcomes of people with CP. In this article, we report the preliminary data of the CP Registry, specifically the demographics, clinical data and functional outcomes of people with CP in Singapore.

\section{METHODS}

\section{Design and study population}

Participation was voluntary. Cases were identified from outpatient clinics, therapy sessions and inpatient admissions at KK Women's and Children's Hospital (KKH) and National University Hospital (NUH), the only tertiary paediatric hospitals in Singapore. Participants were also identified from those attending the Cerebral Palsy Alliance Singapore (CPAS), one of the largest local social service organisations that provides educational and therapy services to people with CP. Recruitment materials were also sent to community providers such as private paediatricians, early intervention centres and special schools. Cases were referred to and screened by the study team of therapists and doctors who had undergone standardised training of the case definition, classification and functional outcome assessments.

In the pilot phase, children born in 2011 and later (6 years and younger) were included. From 2019, people with CP born in 1994 and later were included.

The main inclusion criterion for SingCPR is a diagnosis of CP made by a paediatrician. Our case definition of $\mathrm{CP}$ contains 5 key elements common to the definitions published by Bax, ${ }^{15}$ Mutch $^{16}$ and Rosenbaum, ${ }^{1}$ as adopted by the Surveillance of Cerebral Palsy in Europe ${ }^{2}$ and Australian Cerebral Palsy Register. ${ }^{4}$

Under our definition, cerebral palsy:

(1) is an umbrella term for a group of disorders

(2) is a condition that is permanent but not unchanging

(3) involves a disorder of movement and/or posture and of motor function

(4) is due to a non-progressive interference, lesion, or abnormality

(5) the interference, lesion, or abnormality originates in the immature brain

Based on information from the hospital medical records, $\mathrm{CP}$ was sub-divided into 2 categories: pre/perinatal $\mathrm{CP}$ (defined as an injury to the developing brain throughout pregnancy and the first 28 completed days after birth) and post-neonatal $\mathrm{CP}$ (defined as an injury to the developing brain occurring after 28 days of life and before 3 years of age). Under pre/perinatally acquired $\mathrm{CP}$, data collected for risk factors included: (1) gestation (prematurity was defined as birth that occurs less than 37 completed weeks of gestation); (2) birth weight; (3) small for gestational age; (4) meconium-stained liquor; (5) mode of delivery; (6) multiple births; (7) neonatal encephalopathy; (8) intrauterine infection; (9) congenital anomaly; and (10) unknown. Neonatal encephalopathy is defined as a clinical syndrome in an infant born at or beyond 35 weeks of gestation, manifested by a subnormal level of consciousness or seizures, and often accompanied by difficulty with initiating and maintaining respiration, and depression of tone and reflexes. Under post-neonatally acquired $\mathrm{CP}$, causes consisted of infection, head trauma, cardiovascular accident, anoxic brain injury and others.

Those with progressive neurological conditions as the sole aetiology of their abnormal neurology were excluded, as were people with hypotonia but no other neurological signs, risk factors or abnormal brain imaging.

Clinical data and comorbidities were obtained through hospital medical records. Detailed information on demographics, service utilisation and quality-of-life measures were collected via a standardised questionnaire administered to the family by a study member. Qualityof-life measures included screening questions on general well-being and function using the World Health Organization International Classification of Functioning, Disability and Health, Children and Youth version (ICF-CY) in the domains of Body Structure/Function, Activities and Participation, and Environmental factors. ${ }^{17}$ Levels of ICF-CY impairment was graded according to frequency, intrusiveness or severity from 0 (no impairment/ difficulty) to 4 (complete/ constant 


\section{CLINICAL IMPACT}

\section{What is New}

- The new cerebral palsy (CP) registry in Singapore provides objective data on the causes, functional outcomes and healthcare utilisation of people with $\mathrm{CP}$.

- Pre/perinatally acquired CP accounted for majority of cases, with prematurity as the main risk factor.

- Almost half of participants in this registry suffer from severe motor functional impairment.

\section{Clinical Implications}

- Optimisation of pre- and perinatal care to prevent and manage prematurity, together with early diagnosis and intervention, is important to reduce the incidence, severity and lifelong burden of $\mathrm{CP}$ in Singapore.

impairment/ difficulty/ intensity totally disruptive). ${ }^{18}$ Functional outcome scales including the Gross Motor Function Classification System (GMFCS), ${ }^{19}$ Manual Ability Classification System (MACS), ${ }^{20}$ Eating and Drinking Ability Classification System (EDACS) ${ }^{21}$ and Communication Function Classification System (CFCS), ${ }^{22}$ were assessed by healthcare providers at the respective institutions. The diagnosis of $\mathrm{CP}$ was verified by the study team based on hospital medical records, prior to the final registration and data entry. All data were entered into REDCap, a secure web-based database platform.

\section{Statistical analysis}

For ordinal outcome measures, Wilcoxon Signed-rank Test was used with results presented as frequencies and percentages. For outcome measures with continuous scores, paired t-tests were performed with results in mean and standard deviations. Statistical significance was indicated by a $P$ value $<0.05$. Statistical analysis was conducted with SPSS Statistics software version 19 (IBM Corp, Armonk, US).

\section{Ethics}

Ethics approval for the study was obtained from the SingHealth Institution Review Board (CIRB number: 2016/2266). Informed consent was obtained from all caregivers/participants in accordance with the review board.

\section{RESULTS}

A total of 153 participants were identified during a 31-month period from 1 September 2017 to 31 March 2020. Of these, 2 were excluded for not fulfilling the diagnostic criteria for $\mathrm{CP}$, leaving 151 participants for analysis.

Of the 151 participants, 106 (70.2\%) were males, with a median age of 6.2 years old at recruitment (range 1.2-24.3 years old) and majority were of Chinese ethnicity (Table 1). Ninety (59.6\%) were diagnosed in the first 2 years of life and $117(77.5 \%)$ before 3 years old. Ninety-six of all participants (63.6\%) had a brain magnetic resonance imaging (MRI). The most common abnormal MRI finding was white matter injury (49.5\%), including periventricular leukomalacia and periventricular haemorrhage.

Participants with pre/perinatally acquired CP accounted for $89.4 \%$ (135) of all CP, while participants with post-neonatally acquired CP accounted for $10.7 \%$ (16) of the total group. In the pre/perinatally acquired group $(n=135)$, the majority of participants were born preterm $(75.6 \%)$ and required emergency caesarean section $(50.4 \%)$ while $10.4 \%(14 / 135)$ were born small for gestational age (Table 2). In the post-neonatally acquired group $(n=16)$, the most common cause was infection (37.5\%), followed by head trauma $(25.0 \%)$ (Table 3$)$.

\section{Predominant CP motor type and gross motor function}

In terms of the predominant CP motor type, 109 participants $(72.2 \%)$ had a spastic motor type while $42(27.8 \%)$ had dyskinesia (Table 4). None had choreoathetoid or ataxic CP motor types. Among those with pre/perinatally acquired $\mathrm{CP}, 44$ had spastic diplegia, 30 had spastic monoplegia/hemiplegia, 6 had spastic triplegia and 19 had quadriplegia. Dyskinetic CP constituted $26.7 \%$ of those with pre/peri-neonatally acquired $\mathrm{CP}$ and $37.5 \%$ of those with post-neonatally acquired $\mathrm{CP}$.

For gross motor function $(\mathrm{N}=151), 50$ participants were able to walk independently (GMFCS I-II), 33 were able to walk with assistive devices (GMFCS III) and the remaining 68 were wheelchair-dependent (GMFCS IV-V).

\section{Associated co-morbidities}

Of the 151 participants, $63(41.7 \%)$ had visual impairment, $13(8.6 \%)$ had hearing impairment, 38 $(25.2 \%)$ had epilepsy and $38(25.2 \%)$ had cognitive impairment (Intelligence Quotient $\leq 70$ ). In terms of hip surveillance, $117(77.5 \%)$ had at least 1 pelvis 
Table 1. Baseline characteristics for all cerebral palsy $(\mathrm{N}=151)$

\begin{tabular}{|c|c|}
\hline Characteristics & n (\%) \\
\hline \multicolumn{2}{|l|}{ Sex } \\
\hline Male & $106(70.2)$ \\
\hline Female & $45(29.8)$ \\
\hline \multicolumn{2}{|l|}{ Race } \\
\hline Chinese & $91(60.3)$ \\
\hline Malay & $31(20.5)$ \\
\hline Indian & $17(11.3)$ \\
\hline Other & $12(7.9)$ \\
\hline Age at recruitment, median (range), years & $6.2(1.2-24.3)$ \\
\hline \multicolumn{2}{|l|}{ Age at diagnosis } \\
\hline $0-6$ months & $22(14.6)$ \\
\hline $7-12$ months & $25(16.6)$ \\
\hline $13-24$ months & $44(29.1)$ \\
\hline $25-36$ months & $27(17.9)$ \\
\hline $37-48$ months & $13(8.6)$ \\
\hline $49-60$ months & $6(4.0)$ \\
\hline Age $>5$ years & $8(5.3)$ \\
\hline Not stated & $6(4.0)$ \\
\hline \multicolumn{2}{|l|}{ MRI brain finding $(n=101)^{a}$} \\
\hline Normal & $10(9.9)$ \\
\hline White matter injury (PVH, PVL) & $50(49.5)$ \\
\hline Diffuse cortical injury & $15(14.9)$ \\
\hline Focal cortical injury & $7(6.9)$ \\
\hline Basal ganglia pattern & $8(7.9)$ \\
\hline Malformation & $9(8.9)$ \\
\hline Missing information & $2(2.0)$ \\
\hline
\end{tabular}

MRI: magnetic resonance imaging; PVH: periventricular haemorrhage; PVL: periventricular leukomalacia

a Of 96 participants with MRI brain, 5 had 5 or more dominant MRI findings

X-ray, of whom $24(20.5 \%)$ had hip subluxation and $8(6.8 \%)$ had hip dislocation (Table 4$)$.

\section{Other functional outcomes}

For hand function, 69 (45.7\%) participants were able to handle most objects easily (MACS I-II), 29 (19.2\%) could handle objects independently with modified activities (MACS III) while $53(35.1 \%)$ needed continuous or total assistance (MACS IV-V) (Table 4).
Table 2. Characteristics and risk factors of pre/perinatally acquired cerebral palsy $(\mathrm{n}=135)$

\begin{tabular}{|ll}
\hline Characteristics & $\mathbf{n}(\mathbf{\%})$ \\
\hline Gestational age (weeks) & \\
\hline $23-27$ & $33(24.4)$ \\
\hline $28-31$ & $39(28.9)$ \\
\hline $32-36$ & $30(22.2)$ \\
\hline$\geq 37$ & $33(24.4)$ \\
\hline Birth weight (grams) & \\
\hline$<1000$ & $28(20.7)$ \\
\hline $1000-1499$ & $36(26.7)$ \\
\hline $1500-2499$ & $41(30.3)$ \\
\hline$>2500$ & $30(22.3)$ \\
\hline Mode of delivery & \\
\hline Normal vaginal delivery & $43(31.8)$ \\
\hline Assisted vaginal delivery & $14(10.4)$ \\
\hline Elective caesarean section & $10(7.4)$ \\
\hline Emergency caesarean section & $68(50.4)$ \\
\hline Plurality & $22(6.3)$ \\
\hline Singleton & 0 \\
\hline Twins & $105(77.8)$ \\
\hline Triplets & $27(20.0)$ \\
\hline Risk factors & $3(2.2)$ \\
\hline Meconium-stained liquor & $4(3.0)$ \\
\hline Congenital anomaly & $16(11.9)$ \\
\hline Neonatal encephalopathy & \\
\hline Untrauterine infection & \\
\hline
\end{tabular}

For feeding, most participants were on oral feeding while $19(12.7 \%)$ were on tube feeding (EDACS V) of which, 10 had undergone gastrostomy and fundoplication. Approximately half the participants $(70 / 149,47.0 \%)$ were mostly effective in everyday communication (CFCS I-II) while 60/147 (40.2\%) had inconsistent and limited communication even with familiar partners (CFCS IV-V).

\section{Healthcare utilisation}

Most participants $(102,67.5 \%)$ had frequent medical follow-up ( $\geq 4 /$ year). Only a third $(52,34.4 \%)$ had dental care in the past year. More than half of Singaporeans/ 
permanent residents $(80 / 140,57.9 \%)$ attended or were attending community early intervention programmes, while the rest received or were receiving hospital-based

\begin{tabular}{ll} 
Table 3. Post-neonatal causes (n=16) & \\
\hline Causes & $\mathbf{n}(\mathbf{\%})$ \\
\hline Infection & $6(37.5)$ \\
\hline $\begin{array}{l}\text { Group B Streptococcus meningoencephalitis/ non- } \\
\text { meningoencephalitis with bacteraemia }\end{array}$ & 2 \\
\hline $\begin{array}{l}\text { Streptococcus pneumoniae meningoencephalitis } \\
\text { with bacteraemia }\end{array}$ & 1 \\
\hline HSV type 2 meningoencephalits & 2 \\
\hline Aseptic meningitis with hydrocephalus & 1 \\
\hline Head trauma & $4(25.0)$ \\
\hline Non-accidental injury & 3 \\
\hline Road traffic accident & 1 \\
\hline Cardiovascular accident & $2(12.5)$ \\
\hline Event resulting in hypoxia & $2(12.5)$ \\
\hline Other post-neonatal event & $2(12.5)$ \\
\hline
\end{tabular}

or private therapies. In terms of equipment, 86 of the total participants $(57.0 \%)$ had ankle-foot-orthoses, $31(20.5 \%)$ used walkers or gait trainers, and 17 of $100(17.0 \%)$ participants in GMFCS III-V owned standing frames.

\section{Quality of life outcomes}

Most participating parents $(102,67.5 \%)$ perceived their child to be happy or very happy in general at the point of assessment. The majority of participants reported no or little problem with sleep or pain while $9(6.0 \%), 8$ $(5.3 \%)$ and $5(3.3 \%)$ complained of difficulty in onset of sleep, difficulty in maintaining sleep and generalised pain, respectively. Sleep disturbances and generalised pain were associated with higher GMFCS levels $(P<0.05$ and $P=0.04$, respectively).

\section{DISCUSSION}

This first SingCPR report provided data on the demographics, clinical profiles and functional outcomes of people with CP in Singapore. In our Registry, prematurity is a major risk factor of pre/perinatally

Table 4. Predominant cerebral palsy motor type, functional motor severity classifications and comorbidities

\begin{tabular}{|c|c|c|c|}
\hline & $\begin{array}{l}\text { All CP } \\
\mathrm{n}(\%) \\
(\mathrm{N}=\mathbf{1 5 1})\end{array}$ & $\begin{array}{l}\text { Pre/perinatally acquired CP } \\
\mathrm{n}(\%) \\
(\mathrm{n}=135)\end{array}$ & $\begin{array}{l}\text { Post-neonatally acquired CP } \\
\mathrm{n}(\%) \\
(\mathrm{n}=16)\end{array}$ \\
\hline \multicolumn{4}{|c|}{ Predominant CP motor type and subtype } \\
\hline Spastic & $109(72.2)$ & $99(73.3)$ & $10(62.5)$ \\
\hline $\begin{array}{l}\text { Monoplegia/ } \\
\text { Hemiplegia }\end{array}$ & $35(32.1)$ & $30(30.3)$ & $5(50.0)$ \\
\hline Diplegia & $45(41.2)$ & $44(44.4)$ & $1(10.0)$ \\
\hline Triplegia & $6(5.5)$ & $6(6.0)$ & 0 \\
\hline Quadriplegia & $23(21.1)$ & $19(19.3)$ & $4(40.0)$ \\
\hline Dyskinetic & $42(27.8)$ & $36(26.7)$ & $6(37.5)$ \\
\hline Ataxic & 0 & 0 & 0 \\
\hline \multicolumn{4}{|l|}{ GMFCS } \\
\hline I-II & $50(33.1)$ & $49(36.3)$ & $1(6.2)$ \\
\hline III & $33(21.9)$ & $29(21.5)$ & $4(25.0)$ \\
\hline IV-V & $68(45.0)$ & $57(42.2)$ & $11(68.8)$ \\
\hline \multicolumn{4}{|l|}{ MACS } \\
\hline I-II & $69(45.7)$ & $67(49.6)$ & $2(12.5)$ \\
\hline III & $29(19.2)$ & $25(18.5)$ & $4(25.0)$ \\
\hline IV-V & $53(35.1)$ & $43(31.8)$ & $10(62.5)$ \\
\hline \multicolumn{4}{|l|}{ Comorbidities } \\
\hline Visual impairment & $63(41.6)$ & $57(42.2)$ & $6(37.5)$ \\
\hline Hearing impairment & $13(8.6)$ & $11(8.1)$ & $2(12.5)$ \\
\hline Epilepsy & $38(25.2)$ & $30(22.2)$ & $8(50.0)$ \\
\hline Cognitive impairment & $38(25.2)$ & $32(23.7)$ & $6(37.5)$ \\
\hline
\end{tabular}

CP: cerebral palsy; GMFCS: Gross Motor Function Classification System; MACS: Manual Ability Classification System 
acquired $\mathrm{CP}$ while infection is the most common post-neonatal cause. Spastic CP is the predominant CP motor type. Almost half were in severe impairment groups (GMFCS levels IV-V).

Compared with established $\mathrm{CP}$ registries from other developed countries, we share similar findings of predominant male gender, age of diagnosis typically by 2 years of age and a higher proportion of pre- or perinatally acquired CP. In the group with pre- or perinatally acquired $\mathrm{CP}$, prematurity and the need for emergency caesarean section were the 2 most common risk factors. While recent literature has shown that there was a decreasing prevalence of $\mathrm{CP}$ in moderately preterm as well as moderately and very low birth weight (VLBW) infants, we found preterm birth and birth weight $<2,500 \mathrm{~g}$ in almost two-thirds of our study population, compared with $40-50 \%$ in Western countries. ${ }^{2,4,23,24}$ This figure is consistent with reported Asian data of $70 \%$ in Okinawa, Japan and $60 \%$ in South Korea. ${ }^{6,7}$ This is likely related to our increasing rates of premature birth and decreasing mortality rate of VLBW in Singapore. ${ }^{25}$ Our robust surveillance and follow-up programme in the VLBW cohort has likely also contributed to the high proportion of $\mathrm{CP}$ associated with prematurity. ${ }^{26}$

Among participants with post-neonatally acquired $\mathrm{CP}$, infection and non-accidental head trauma were the commonest aetiologies. Some of these infections were potentially preventable causes. Vaccination against pneumococcal disease was recently included as one of the nationally recommended vaccines, with enhanced subsidy in Singapore. We hope that this will further reduce the risk of pneumococcal meningoencephalitis that may result in severe neurological sequelae. In terms of non-accidental head injury in infants, there needs to be further education for parents and carers in the risks of shaking a baby as well as identifying families who may require additional support.

In our Registry, spasticity was the most common dominant motor type, similar to other registries. However, unlike in Canada and in Australia, spastic diplegia is more common than spastic hemiplegia in Singapore. ${ }^{4,24}$ This is related to the higher rate of premature birth, low birth weight and associated periventricular leukomalacia in our Registry. ${ }^{27,28}$

Similar to registries from Europe and Canada, we classified CP into 3 motor types: spastic, dyskinetic and ataxic. ${ }^{29}$ In our Registry, dyskinetic CP represent a larger proportion $(27.8 \%$ overall) as compared to $7-12 \%$ in Europe and Australia. ${ }^{4,30,31}$ This may be related to specialist recognition of dyskinesia in our hospitalbased cohort. Separately, due to a rigorous national neonatal hyperbilirubinemia screening and treatment programme, we had no local choreoathetoid $\mathrm{CP}$ associated with kernicterus.

We recorded no cases of hypotonic or ataxic $\mathrm{CP}$ in our Registry while other registries reported $<10 \%$ of hypotonic or ataxic CP.,13,32 In our case definition, hypotonic children with no other neurological sign, risk factor or abnormal brain imaging were excluded and we performed metabolic and genetic investigations in children with hypotonia and/or early-onset ataxia in line with recommendations. ${ }^{34}$ Furthermore, the description and definition of ataxic $\mathrm{CP}$ is generally lacking. A high proportion of people with ataxic $\mathrm{CP}$ can have an incorrect initial diagnosis. ${ }^{33}$ Thus, it is rare to make a diagnosis of ataxic or hypotonic $\mathrm{CP}$ in our clinical practice.

With regards to comorbidities, we reported similar findings in epilepsy, visual impairment, cognitive impairment and hip displacements as most other registries. ${ }^{4,13,23,24,31,32}$ In terms of gross motor function, our Registry reported higher proportions (45\%) of participants with severe impairments (GMFCS IV-V, $25-28 \%$ in established CP registries). ${ }^{4,24}$ This may be explained by our Registry being hospital- and centrebased, as compared to other community registries.

Unique to our Registry, we collected comprehensive data on measures related to quality of life. It is heartening to know that most parents perceived their child to be happy in general and majority of the participants had no or little problem with sleep or pain. However, those with higher GMFCS levels were more likely to complain of sleep disturbances and pain, comparable with studies that indicated that sleep disorders are positively associated with impaired gross motor function. ${ }^{35,36}$ Participants with higher GMFCS were more likely to experience pain from muscle spasms, hip dislocation and difficulties in changing sleep positions, which worsen sleep disturbances. ${ }^{37}$ There is a pressing need to look into the practical management of these specific issues to improve the quality of life of these patients.

\section{Strengths and limitations}

The strength of our Registry is active recruitment from the 2 main paediatric hospitals and 1 of the largest CP service providers in Singapore. We also had strict case definitions of $\mathrm{CP}$ and the participants' diagnoses were verified by paediatricians using hospital medical records before final recruitment. Moreover, data in our Registry were extensive and included measures for quality of life and service utilisation. At the same time, information was complete for the motor subtypes and functional classification scales of the participants. 
The main limitation of the Registry comes from likely selection bias, due to the process of voluntary recruitment through tertiary hospitals, increasing the likelihood of recruiting participants with more severe disabilities and missing participants with mild disabilities who are not assessing these services. Such selection bias could have contributed a higher proportion of severe $\mathrm{CP}$ and a lower proportion of hemiplegic $\mathrm{CP}$ in our Registry.

Moving forward, we hope to expand community recruitment so that we could include more participants with less severe disabilities. In addition, the registry has planned for regular review and update of our patients' information. With time, this will allow us to track the burden of the disease locally, to allow policymakers and administrators to better plan health resources allocation and management.

\section{CONCLUSION}

This is the first report of the newly established CP Registry in Singapore that provided objective data on the causes, functional outcomes and healthcare utilisation of people with CP locally. Pre/perinatally acquired $\mathrm{CP}$ accounted for the majority of all cases, with prematurity being the main risk factor. Almost half of the registry is in the severe motor functional impairment groups. Optimisation of pre- and perinatal care to prevent and manage prematurity, together with early diagnosis and intervention, remains an important strategy to reduce the incidence, severity and chronic burden of the condition locally. As the Registry continues to grow in the future, we are confident this work will provide important new insights into our understanding of this common lifelong neurodevelopmental disorder.

\section{Acknowledgements}

The authors would like to acknowledge Ms Latha Kuthy and all staff of Cerebral Palsy Alliance Singapore for their support in setting up the Registry. We thank Ms Su Bing for maintaining the database, Ms Melody Por for recruitment efforts, Dr Dhivya Muthiah and Dr Teo Tong Lin for their inputs in data interpretation, as well as the patients and their families who contributed to the Registry.

\section{REFERENCES}

1. Rosenbaum P, Paneth N, Leviton A, et al. A report: the definition and classification of cerebral palsy April 2006. Dev Med Child Neurol Suppl 2007 Feb;109:8-14.

2. Sellier E, Platt MJ, Andersen GL, et al. Decreasing prevalence in cerebral palsy: a multi-site European population-based study, 1980 to 2003. Dev Med Child Neurol 2016;58:85-92.
3. Oskoui M, Coutinho F, Dykeman J, et al. An update on the prevalence of cerebral palsy: a systematic review and meta-analysis. Dev Med Child Neurol 2013;55:509-19.

4. Report of the Australian Cerebral Palsy Register, Birth Years 1995-2012, 2018. Available at https://cpregister.com/wp-content/ uploads/2019/02/Report-of-the-Australian-Cerebral-Palsy-RegisterBirth-Years-1995-2012.pdf. Accessed on 14 Jan 2021.

5. Goldsmith S, McIntyre S, Smithers-Sheedy H, et al. An international survey of cerebral palsy registers and surveillance systems. Dev Med Child Neurol 2016;58 Suppl 2(Suppl 2):11-7.

6. Yim SY, Yang CY, Park JH, et al. Korean Database of Cerebral Palsy: A Report on Characteristics of Cerebral Palsy in South Korea. Ann Rehabil Med 2017;41:638-49.

7. Touyama M, Touyama J, Toyokawa $\mathrm{S}$, et al. Trends in the prevalence of cerebral palsy in children born between 1988 and 2007 in Okinawa, Japan. Brain Dev 2016;38:792-9.

8. Liu JM, Li S, Lin Q, et al. Prevalence of cerebral palsy in China. Int J Epidemiol 1999;28:949-54.

9. He P, Chen G, Wang Z, et al. Children with motor impairment related to cerebral palsy: Prevalence, severity and concurrent impairments in China. J Paediatr Child Health 2017;53:480-4.

10. Yam WK, Chan HS, Tsui KW, et al. Prevalence study of cerebral palsy in Hong Kong children. Hong Kong Med J 2006;12:180-4.

11. Ibrahim SH, Bhutta ZA. Prevalence of early childhood disability in a rural district of Sind, Pakistan. Dev Med Child Neurol 2013; 55:357-63.

12. Thapa R. Retrospective Descriptive Study of Cerebral Palsy in Nepal. J Autism Dev Disord 2016;46:2285-91.

13. Khandaker G, Muhit M, Karim T, et al. Epidemiology of cerebral palsy in Bangladesh: a population-based surveillance study. Dev Med Child Neurol 2019;61:601-9.

14. Khandaker G, Van Bang N, Dũng TQ, et al. Protocol for hospital based-surveillance of cerebral palsy $(\mathrm{CP})$ in Hanoi using the Paediatric Active Enhanced Disease Surveillance mechanism (PAEDS-Vietnam): a study towards developing hospital-based disease surveillance in Vietnam. BMJ Open 20179;7:e017742.

15. Bax MC. TERMINOLOGY AND CLASSIFICATION OF CEREBRAL PALSY. Dev Med Child Neurol 1964;6:295-7.

16. Mutch L, Alberman E, Hagberg B, et al. Cerebral palsy epidemiology: where are we now and where are we going? Dev Med Child Neurol. 1992 Jun;34(6):547-51.

17. Schiariti V, Tatla S, Sauve K, et al. Toolbox of multiple-item measures aligning with the ICF Core Sets for children and youth with cerebral palsy. Eur J Paediatr Neurol 2017 Mar;21:252-63.

18. Liow NY, Gimeno H, Lumsden DE, et al. Gabapentin can significantly improve dystonia severity and quality of life in children. Eur J Paediatr Neurol 2016;20:100-7.

19. Palisano RJ, Rosenbaum P, Bartlett D, et al. Content validity of the expanded and revised Gross Motor Function Classification System. Dev Med Child Neurol 2008;50:744-50.

20. Eliasson AC, Krumlinde-Sundholm L, Rösblad B, et al. The Manual Ability Classification System (MACS) for children with cerebral palsy: scale development and evidence of validity and reliability. Dev Med Child Neurol 2006;48:549-54.

21. Tschirren L, Bauer S, Hanser C, et al. The Eating and Drinking Ability Classification System: concurrent validity and reliability in children with cerebral palsy. Dev Med Child Neurol 2018;60:611-7. 
22. Hidecker MJ, Paneth N, Rosenbaum PL, et al. Developing and validating the Communication Function Classification System for individuals with cerebral palsy. Dev Med Child Neurol 2011; 53:704-10.

23. Andersen GL, Irgens LM, Haagaas I, et al. Cerebral palsy in Norway: prevalence, subtypes and severity. Eur J Paediatr Neurol 2008;12:4-13.

24. Hadjinicolaou A, Ng P, Ph DX, et al. Is Cerebral Palsy Changing in High Resource Settings? Data From the Quebec Cerebral Palsy Registry. J Child Neurol 2019;34:567-73.

25. Report on Registration of Birth and Deaths 2017, June 2018. Available at https://www.ica.gov.sg/docs/default-source/ica/stats/annual-bdstatistics/stats_2017_annual_rbd_report.pdf. Accessed on 14 Jan 2021.

26. Anand AJ, Sabapathy K, Sriram B, et al. Single Center Outcome of Multiple Births in the Premature and Very Low Birth Weight Cohort in Singapore. Am J Perinatol 2020.

27. Agarwal P, Sriram B, Lim SB, et al. Borderline viability--neonatal outcomes of infants in Singapore over a period of 18 years (19902007). Ann Acad Med Singap 2013;42:328-37.

28. Teo CM, Poon WB, Ho SK. Long-Term Neurodevelopmental Outcomes of Premature Infants in Singapore. Ann Acad Med Singap 2018;47:63-70

29. Surveillance of cerebral palsy in Europe: a collaboration of cerebral palsy surveys and registers. Surveillance of Cerebral Palsy in Europe (SCPE). Dev Med Child Neurol 2000;42:816-24.
30. Préel M, Rackauskaite G, Larsen ML, et al. Children with dyskinetic cerebral palsy are severely affected as compared to bilateral spastic cerebral palsy. Acta Paediatr 2019;108:1850-6.

31. Gincota Bufteac E, Andersen GL, Torstein V, et al. Cerebral palsy in Moldova: subtypes, severity and associated impairments. BMC Pediatr 2018;18:332.

32. Jonsson U, Eek MN, Sunnerhagen KS, et al. Cerebral palsy prevalence, subtypes, and associated impairments: a population-based comparison study of adults and children. Dev Med Child Neurol 2019;61:1162-7.

33. Chen A, Dyck Holzinger S, Oskoui M, et al. Losing a diagnosis of cerebral palsy: a comparison of variables at 2 and 5 years. Dev Med Child Neurol 2020;62:83-8.

34. Dan B. How useful is the diagnosis of ataxic cerebral palsy? Dev Med Child Neurol 2020;62:264.

35. Jacquier D, Newman CJ. Co-sleeping in school-aged children with a motor disability: a comparative population-based study. Dev Med Child Neurol 2017;59:420-6.

36. Petersen S, Francis KL, Reddihough DS, et al. Sleep problems and solution seeking for children with cerebral palsy and their parents. J Paediatr Child Health 2020;56:1108-13.

37. Eriksson E, Hägglund G, Alriksson-Schmidt AI. Pain in children and adolescents with cerebral palsy - a cross-sectional register study of 3545 individuals. BMC Neurol 2020;20:15 